TITLE:

\title{
Will it be possible to generate kidney tissue from induced pluripotent stem cells for regenerative therapy?
}

$\operatorname{AUTHOR}(S):$

Osafune, Kenji

CITATION:

Osafune, Kenji. Will it be possible to generate kidney tissue from induced pluripotent stem cells for regenerative therapy?. Regenerative medicine 2014, 9(1): 9-12

ISSUE DATE:

2014-01

URL:

http://hdl.handle.net/2433/196855

RIGHT:

(C) 2014 Future Medicine Ltd.; Publisher permitted to post the version of record. 


\title{
Will it be possible to generate kidney tissue from induced pluripotent stem cells for regenerative
} therapy?

\begin{abstract}
"Regenerative medicine strategies are highly anticipated for chronic kidney disease, since patients with chronic kidney disease never recover their renal function, and there are no radical treatments besides renal transplantation."
\end{abstract}

\section{KEYWORDS: bioartificial kidney = biological scaffold = blastocyst complementation = decellularization $\approx$ ESC $\approx$ iPSC $\approx$ kidney tissue $\approx$ self-organization $\approx$ tissue engineering - vascularization}

Chronic kidney disease (CKD) is increasingly recognized as a global public health problem. The increased prevalence of CKD has led to a rise in the number of dialysis patients, and is associated with elevated morbidity and mortality due to the increased risk of cardiovascular disease [1]. Regenerative medicine strategies are highly anticipated for CKD, since patients with CKD never recover their renal function, and there are no radical treatments besides renal transplantation. With this as the goal, vigorous efforts have been made for the directed differentiation of mouse embryonic stem cells (mESCs) $[2,3]$ into kidney lineage cells, while research using human ESCs [4] or induced pluripotent stem cells (hiPSCs) $[5,6]$ has not been fully developed [7].

By mimicking signals that occur during embryonic development, to the extent that they are known, a stepwise protocol was explored to differentiate pluripotent stem cells (PSCs) into clinically useful cell types in adult organs. Currently, most of the studies aiming to generate renal cells from PSCs have also adopted the strategy of mimicking renal development [7]. The kidneys are derived from an early embryonic germ layer, the intermediate mesoderm (IM) that is located between the lateral and paraxial mesoderms [8]. In vertebrates, the IM successively develops three kidneys: the pronephros, mesonephros and metanephros. The three kidneys are similar in that they consist of a basic functional unit, the nephron, although the number of nephron differs among the kidneys. The mammalian adult kidney, metanephros, is formed by the reciprocal interaction between two tissues derived from the IM, the metanephric mesenchyme and ureteric bud. The ureteric bud induces the metanephric mesenchyme to differentiate into nephrons and interstitium. The nephron progenitors in metanephric mesenchyme differentiate into epithelia constituting glomeruli, proximal or distal renal tubules, or Henle's loop. The metanephric mesenchyme may contain at least two other precursor populations, in addition to the epithelial progenitors: vascular precursor cells that give rise to vascular and glomerular endothelia and vascular smooth muscle cells; and stromal precursors that eventually differentiate into interstitial cells within the adult kidney. The ureteric bud elaborates the lower urinary tract system, from the collecting ducts through the renal pelvis and ureters, to a part of the urinary bladder [8]. Most of the mechanisms underlying the lineage commitment, by which the IM gives rise to the metanephric mesenchyme and ureteric bud, and how the two precursors differentiate into adult renal cell types, are unknown. Further examinations will be required to elucidate the mechanisms, which will eventually help reproduce the tissues in vitro from PSCs.

Our group has recently published a report on the highly efficient differentiation of human PSCs into IM cells [9]. These human IM cells have the developmental potential to further differentiate into adult and embryonic renal cells, such as glomerular podocytes, proximal renal tubular cells and ureteric bud cells. Furthermore, they can form 3D renal tubular structures when co-cultured with mouse metanephric cells in organ culture settings. However, the efficiency of tubule formation is very low, at around $5 \%$ of the organ cultures. We reasoned that this observed low efficiency resulted from the developmental immaturity of the IM cells. Therefore, our group is currently developing efficient differentiation protocols from human PSC-derived IM cells into the two kidney

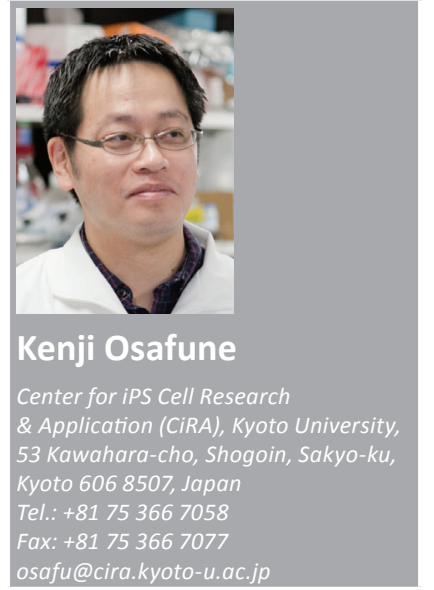


precursors, metanephric mesenchyme and ureteric bud, and adult renal cell types, including glomerular podocytes, renal tubular cells and collecting duct cells.

After the successful efficient induction of kidney lineage cells from PSCs, the next steps toward the development of renal regenerative medicine would include the generation of functional renal tissues that are integrated with the vasculature, that filter blood and produce urine. However, precisely how these functional renal tissues can be made from PSCs remains a question to be answered in the near future.

One of the clues to the answer is the potential of PSC-derived progenitor cells to self-organize, by which ordered tissue structures are spontaneously formed through complex collective cell behaviors without obvious predetermined patterns. Sasai et al. have demonstrated that the PSC-derived neural precursors can self-organize and spontaneously form multilayered cerebral cortical tissues, optic cup structures with multilayered neural retina and adenohypophysis tissues $[10,11]$. It has also been known since the 1950s that metanephric tissues have the potential to self-organize spontaneously to form 3D renal structures in organ culture settings that contain glomeruli, renal tubules and collecting ducts, but they are deprived of the vasculature in culture, resulting in the formation of avascular glomeruli [12].

Although it may be possible to make renal tissues by utilizing the potential of PSC-derived precursors to self-organize, the next question is how to make them vascularized. One answer can be found in the literature, and is the utilization of an in vivo microenvironment which supplies the implanted tissues with vasculature. When combined with the potential for self-organization of the renal progenitors, the environmental cues might help them form vascularized tissues. Hammerman et al. and Dekel et al. demonstrated the formation of vascularized renal tissue from metanephroi inside the rodent body $[13,14]$. When mouse, rat, porcine or even human early gestational metanephroi are transplanted into the kidney subcapsule or the abdominal cavity of immunodeficient mice, they survive, grow, undergo complete nephrogenesis and form functional organs able to produce urine by being integrated with the host vasculature and forming vascularized glomeruli. Although it was difficult to connect the vascularized donor nephrons to the lower urinary tract towards the urinary bladder of the host animals, the reports did succeed in generating vascularized kidney tissues.
These reports used metanephroi from experimental animals and humans. However, it was unclear whether it may be possible to generate functional tissues from the PSC-derived cells inside the animal body. Taniguchi et al. have recently succeeded in generating vascularized and functional human liver tissue from hiPSCs using a mouse cranial window model [15]. They first formed liver bud tissues in vitro from hiPSC-derived hepatoblasts, human mesenchymal stem cells and human endothelial cells (human umbilical vein endothelial cells) by recapitulating embryonic liver development, and then transplanted the liver buds into a preformed cranial window of an immunodeficient mouse. The human liver buds were shown to connect to the host vasculature and elaborate functional hepatic cord-like structures that could perform liver-specific functions, such as protein production and human-specific drug metabolism. Similar strategies could be used for the generation of vascularized and functional renal tissues from PSC-derived renal progenitors inside the rodent body.

\section{"Continued efforts to elucidate the mechanisms underlying the mesoderm formation and development of renal lineage and nephron specification are needed. Such investigations are indispensable for establishing methods to induce directed differentiation from pluripotent stem cells into renal lineage cells."}

Other strategies to generate vascularized and functional tissues or organs would include the utilization of a biological scaffold for the cells to form a 3D architecture. Ross et al. decellularized rat kidneys by detergent perfusion and reseeded them with undifferentiated mESCs, resulting in the differentiation of mESCs into renal cells in the scaffold [16]. Ott et al. have recently published a report showing that decellularized rat kidneys were repopulated with rat neonatal kidney cells and human umbilical vein endothelial cells, and that the regenerated kidneys showed urine production both in vitro and after transplantation in vivo [17]. Decellularizing patient-derived kidneys and refilling them with the renal precursors and vascular progenitors differentiated from patient-derived iPSCs might lead to the reconstruction of $3 \mathrm{D}$ whole kidneys that can be used for autologous renal transplantation.

On the other hand, Nakauchi et al. demonstrated that entire organs can be generated by 
using a method involving interspecific blastocyst complementation [18]. They injected mouse and rat ESCs/iPSCs into $\mathrm{Pdx}^{(--)}$(pancreatogenesis-disabled) rat and mouse blastocysts, respectively, and generated an almost entirely PSC-derived pancreas interspecifically. Similar experiments have already been performed to generate entire kidneys by injecting mouse PSCs into the blastocysts of Sall1 $1^{(--)}$mice, which lose parts of their renal tissues derived from the metanephric mesenchyme and die of renal insufficiency after birth [19]. The resultant kidneys showed the chimerism of donor-derived mesenchyme and stroma lineage renal cells and host-derived vascular and ureteric bud lineage cells. These approaches hold tremendous promise for generating functional whole kidneys, and the generation of genetically engineered host animals that lose all the components of kidneys, including the vasculature, will be required for further analyses.

The tissue engineered bioartificial kidney combining a synthetic membrane hemofiltration device substituting for the small soluble clearance function of renal glomeruli and a renal tubule cell therapy device in an extracorporeal blood perfusion circuit is also among the functional renal tissue alternatives [20]. The therapy compensates for the metabolic, immunoregulatory and endocrinologic functions of the kidney lost in both acute and chronic renal failure that cannot be adequately treated with conventional dialysis therapy. Although Humes $e t$ al. already succeeded in establishing the propagation methodology for renal tubular epithelial cells from human donor kidneys for the device [21], renal tubular cells generated from PSCs might be another source of cells for the treatment, which might be autologous and more desirable in terms of immunological aspects and infections.

The development of regenerative medicine strategies for CKD has just started. The research on the directed differentiation of PSCs into renal lineages is currently underway. Continued efforts to elucidate the mechanisms underlying the mesoderm formation and development of renal lineage and nephron specification are needed. Such investigations are indispensable for establishing methods to induce directed differentiation from PSCs into renal lineage cells. The cues leading to the generation of functional renal tissues from PSCs discovered so far include the self-organization potential of progenitor cells, in vivo environment promoting a vasculature supply, the use of a biological scaffold and the use of tissue engineering technologies. Collective and integrative research focusing on developmental biology, stem cell biology and tissue-engineering will be required to achieve this goal.

\section{Financial \& competing interests disclosure}

The author has no relevant affiliations or financial involvement with any organization or entity with a financial interest in or financial conflict with the subject matter or materials discussed in the manuscript. This includes employment, consultancies, honoraria, stock ownership or options, expert testimony, grants or patents received or pending, or royalties.

No writing assistance was utilized in the production of this manuscript.

\section{References}

1 Go AS, Chertow GM, Fan D et al. Chronic kidney disease and the risks of death, cardiovascular events, and hospitalization. N. Engl. J. Med. 351(13), 1296-1305 (2004).

2 Evans MJ, Kaufman MH. Establishment in culture of pluripotential cells from mouse embryos. Nature 292(5819), 154-156 (1981).

3 Martin GR. Isolation of a pluripotent cell line from early mouse embryos cultured in medium conditioned by teratocarcinoma stem cells. Proc. Natl Acad. Sci. USA 78(12), 7634-7638 (1981).

4 Thomson JA, Itskovitz-Eldor J, Shapiro SS et al. Embryonic stem cell lines derived from human blastocysts. Science 282(5391), 1145-1147 (1998)

5 Takahashi K, Tanabe K, Ohnuki M et al. Induction of pluripotent stem cells from adult human fibroblasts by defined factors. Cell 131(5), 861-872 (2007).

6 Yu J, Vodyanik MA, Smuga-Otto K et al. Induced pluripotent stem cell lines derived from human somatic cells. Science 318(5858), 1917-1920 (2007).

7 Osafune K. iPS cell technology-based research for the treatment of diabetic nephropathy. Semin. Nephrol. 32(5), 479-485 (2012).

8 Dressler GR. Advances in early kidney specification, development and patterning. Development 136(23), 3863-3874 (2009).

9 Mae S, Shono A, Shiota F et al. Monitoring and robust induction of nephrogenic intermediate mesoderm from human pluripotent stem cells. Nat. Commun. 4, 1367 (2013).
10 Eiraku M, Tanaka N, Ishibashi $\mathrm{H}$ et al. Self-organizing optic-cup morphogenesis in three-dimensional culture. Nature 472(7341), 51-56 (2011).

11 Sasai Y. Cytosystems dynamics in selforganization of tissue architecture. Nature 493(7432), 318-326 (2013).

12 Grobstein C. Inductive epitheliomesenchymal interaction in cultured organ rudiments of the mouse metanephros. Science 118(3054), 52-55 (1953).

13 Rogers SA, Lowell JA, Hammerman NA et al. Transplantation of developing metanephroi into adult rats. Kidney Int. 54(1), 27-37 (1998).

14 Dekel B, Burakova T, Arditti FD et al. Human and porcine early kidney precursors as a new source for transplantation. Nat. Med. 9(1), 53-60 (2003). 
15 Takebe T, Sekine K, Enomura M et al. Vascularized and functional human liver from an iPSC-derived organ bud transplant. Nature 499(7459), 481-484 (2013).

16 Ross EA, Williams MJ, Hamazaki T et al. Embryonic stem cells proliferate and differentiate when seeded into kidney scaffolds. J. Am. Soc. Nephrol. 20(11), 2338-2347 (2009).

17 Song JJ, Guyette JP, Gilpin SE et al. Regeneration and experimental orthotopic transplantation of a bioengineered kidney. Nat. Med. 19(5), 646-651 (2013).

18 Kobayashi T, Yamaguchi T, Hamanaka S et al. Generation of rat pancreas in mouse by interspecific blastocyst injection of pluripotent stem cells. Cell 142(5), 787-799 (2010).

19 Usui J, Kobayashi T, Yamaguchi T et al. Generation of kidney from pluripotent stem cells via blastocyst complementation. Am. J. Pathol. 180(6), 2417-2426 (2012).
20 Humes HD, Buffington DA, MacKay SM, Funke AJ, Weitzel WF. Replacement of renal function in uremic animals with a tissueengineered kidney. Nat. Biotechnol. 17(5), 451-455 (1999).

21 Humes HD, Buffington D, Westover AJ, Roy S, Fissell WH. The bioartificial kidney: current status and future promise. Pediatr. Nephrol. doi:10.1007/s00467-013-2467-y (2013) (Epub ahead of print). 
Reproduced with permission of the copyright owner. Further reproduction prohibited without permission. 